\title{
SHiP: a new facility with a dedicated detector to search for new long-lived neutral particles
}

\author{
Elena Graverini*, on behalf of the SHiP collaboration \\ Universität Zürich $(\mathrm{CH})$ \\ E-mail: elena.graveriniecern.ch
}

\begin{abstract}
SHIP is a new fixed target experiment whose Technical Proposal has been recently submitted to the CERN SPS Committee. In its initial phase, the $400 \mathrm{GeV}$ proton beam extracted from the SPS will be dumped on a heavy target with the aim of integrating $2 \times 10^{20}$ pot in 5 years. A dedicated detector, based on a long empty decay volume followed by a spectrometer and particle identification detectors, will allow probing a variety of models with light long-lived exotic particles and masses below $\mathscr{O}(10) \mathrm{GeV} / c^{2}$. The main focus will be the physics of the so-called Hidden Portals, i.e. search for Dark Photons, light scalars and pseudo-scalars, and Heavy Neutrinos. The sensitivity to Heavy Neutrinos will allow for the first time to probe, in the mass range between the kaon and the $D$ meson mass, a coupling range for which Baryogenesis and active neutrino masses could also be explained. Direct detection of light and long-lived SUSY particles, such as RPV neutralinos and pseudo-Dirac gauginos could also be performed in an unexplored parameter range.
\end{abstract}

The European Physical Society Conference on High Energy Physics

22-29 July 2015

Vienna, Austria

${ }^{*}$ Speaker. 


\section{Introduction and physics case}

The Standard Model (SM) describes all known microscopic physics phenomena with great precision, apart from the observed neutrino oscillations. The existence of Dark Matter and the matter-antimatter asymmetry in the Universe give additional evidence that the SM is not a complete theory. In particular, Dark Matter is explained naturally in models with an hidden or secluded sector. An example is the $v$ MSM model [4] where three right-handed neutrinos, particles also known as sterile or Majorana neutrinos or Heavy Neutral Leptons (HNLs), are added to the particle content of the Standard Model, addressing all the known deficiencies of the SM. One of the new states is a dark matter candidate with mass in the keV region. The other two are degenerate in mass, in the $\mathrm{MeV}-\mathrm{GeV}$ range, and are responsible for the masses of SM neutrino through the seesaw mechanism. These two massive states can also be responsible for the matter-antimatter asymmetry of the Universe, generated through leptogenesis.

SHiP is a newly proposed general purpose fixed target experiment at the CERN SPS accelerator, aiming to look for very weakly interacting long living particles. In the context of sterile neutrinos, it would allow to probe most of the allowed parameter space below a few GeV. In addition to the search for HNLs, for which the experiment was devised, SHiP can test several other models of physics beyond the SM, involving very weakly interacting long-lived particles. Moreover, a dedicated detector for studying tau neutrino properties and nucleon structure functions will be installed alongside with the main detector. A detailed description of the SHiP physics case can be found in [2].

\section{The SHiP experiment}
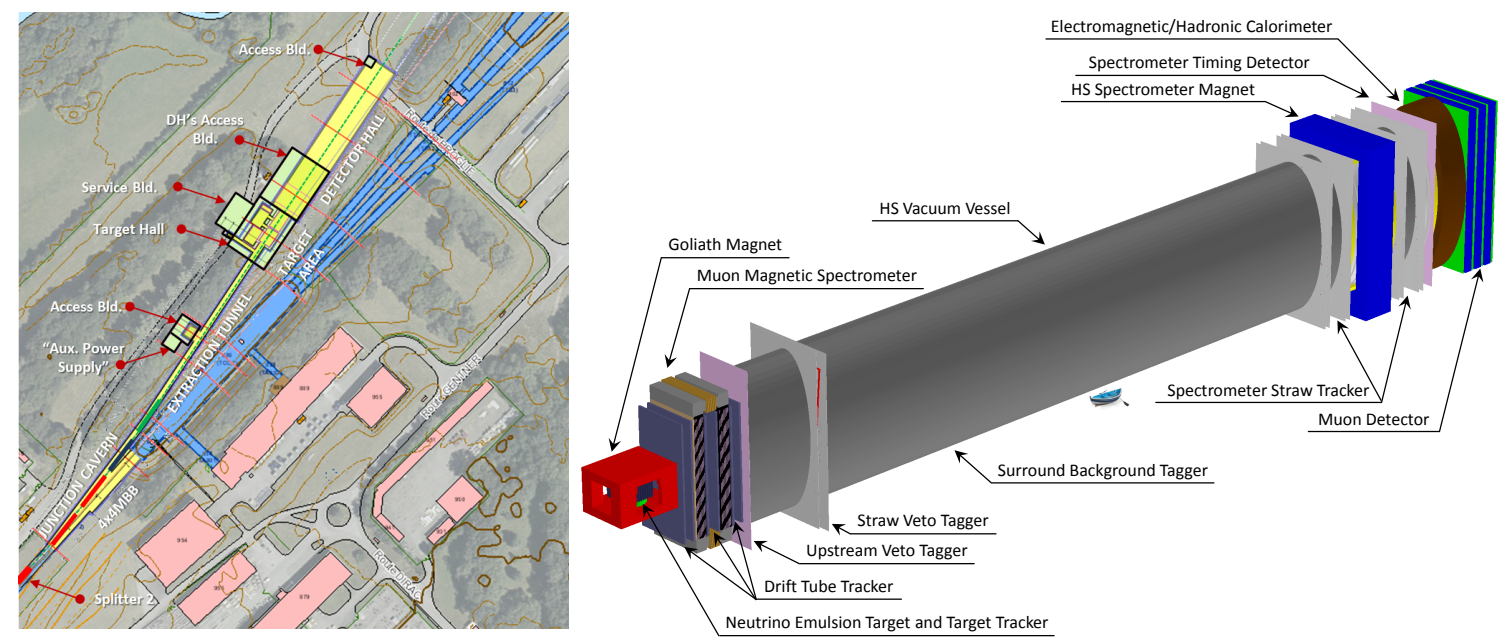

Figure 1: Left: overview ofthe SHiP facility. Right: detailed view of the SHiP tau neutrino and hidden sector detectors (GEANT4).

The SHiP experiment will make use of a new, dedicated beam line branched off the SPS extraction line at the CERN North Area. The proposed location of the beam dump and underground complex (Figure 1) allows for future extensions of the experimental hall. 
The $400 \mathrm{GeV} / \mathrm{c}$ proton beam will be stopped in a heavy target, at a center-of-mass energy $E_{C M}=\sqrt{2 E_{b} m_{p}} \simeq 27 \mathrm{GeV}$. The target must maximize the production of heavy mesons while reducing that of neutrinos and muons to a minimum, and should thus be made of a material with the shortest possible interaction length, for a total size capable of containing the proton shower. A detailed study proved that the desired performance can be achieved with a segmented target made of layers of titanium-zirconium doped molybdenum and of pure tungsten, alternated with thin slits for water cooling. Under nominal SPS conditions, the SPS will deliver $2 \times 10^{20}$ protons on the SHiP target [3].

The target will be followed by a hadron stopper in iron, with the aim of absorbing secondary hadrons and residual non-interacting protons. Muons emerging from the beam dump will be efficiently deflected from the detector fiducial volume by means of an active shielding system based on magnets of alternate polarity, designed on the basis of the expected muon spectrum. Detailed simulations prove the efficiency of this configuration, capable of reducing the flux of muons in the solid angle of the Hidden Sector (HS) detector to an acceptable level in only $48 \mathrm{~m}$ of space.

\subsection{The $v_{\tau}$ detector}

The tau neutrino detector is smaller than the HS detector and can be placed in front of it, still benefitting of the muon-cleared area. It will consist of target made of bricks of laminated lead and emulsions placed in magnetic field, similar to those used by the OPERA experiment [1], followed by a target tracker and by a muon magnetic spectrometer. Details about the $v_{\tau}$ detector and its physics case are given in [6].

\subsection{Hidden Sector detector}

The hidden sector detector will search for evidence of hidden particles decaying in a single large decay volume. This volume is defined by a vacuum vessel with elliptical cross-section of $5 \mathrm{~m}$ width by $10 \mathrm{~m}$ height. The length of the tube is $62 \mathrm{~m}$, consisting of $50 \mathrm{~m}$ of fiducial decay volume and a $12 \mathrm{~m}$ long magnetic spectrometer. Our studies have proved that a level of vacuum of $10^{-6}$ atm is needed to reduce the background due to SM neutrinos to less than one interaction in 5 years of running.

The vessel itself will be instrumented with a background tagger. The $30 \mathrm{~cm}$ of gap inside the double external wall of the decay volume will be filled of liquid scintillator, separated into 863 sections of approximately $1.5 \mathrm{~m}^{2}$ of surface each read out by two large-area photodetectors.

An important source of background is represented by neutral kaons produced in neutrino and muon interactions upstreams of the vacuum vessel. Since the last layer of the muon spectrometer of the $v_{\tau}$ detector does not cover the full HS detector acceptance, another upstream veto tagger is foreseen. This detector will consist of a $4 \times 12 \mathrm{~m}^{2}$ array of plastic scintillator bars.

Background originating in the entrance window of the vacuum vessel is tagged by a straw veto tagger, located in vacuum at $5 \mathrm{~m}$ from the entrance window. The same technology is used for the magnetic spectrometer tracker. The HS spectrometer must be able to reconstruct charged tracks from the decay of hidden particles with maximum efficiency, while also providing accurate measurements of the track momentum and direction of flight. Background rejection in SHiP can deeply benefit of high precision timing, good quality vertices, and of a good resolution in the reconstructed invariant mass and pointing. The tracker will consist of four stations symmetrically 
Table 1: Summary of the main decay modes of hidden particles in various models $(\ell=e, \mu)[3]$.

\begin{tabular}{|cc|}
\hline Models & Final states \\
\hline Neutrino portal, SUSY neutralino & $\ell^{ \pm} \pi^{\mp}, \ell^{ \pm} K^{\mp}, \ell^{ \pm} \rho^{\mp}, \rho^{ \pm} \rightarrow \pi^{ \pm} \pi^{0}$ \\
\hline Vector, scalar, axion portals, SUSY sgoldstino & $\ell^{+} \ell^{-}$ \\
Vector, scalar, axion portals, SUSY sgoldstino & $\pi^{+} \pi^{-}, K^{+} K^{-}$ \\
Neutrino portal, SUSY neutralino, axino & $\ell^{+} \ell^{-} v$ \\
Axion portal, SUSY sgoldstino & $\gamma \gamma$ \\
SUSY sgoldstino & $\pi^{0} \pi^{0}$ \\
\hline
\end{tabular}

arranged around a large aperture dipole magnet providing an integrated field of $0.65 \mathrm{Tm}$. Each station provides two stereo views and two views in $y$, the direction perpendicular to the magnetic field. The R\&D of the straw tracker will benefit from the positive performances recorded in NA62 [5], the only difference being the need for $5 \mathrm{~m}$ long straw tubes.

Outside of the vacuum vessel, a timing detector with a resolution of 100 ps will allow to efficiently tag events originating from random combinations of two muons. Two technologies are currently under investigation, both capable of achieving the required performance: plastic scintillator bars and multi-gap resistive plate chambers (MRPC).

The electromagnetic calorimeter will identify electrons, photons and neutral pions and provide precise energy measurements by means of a shashlik structure of interlaced lead and plastic scintillator planes read out by plastic wavelength-shifting fibers. It will be followed by a hadronic calorimeter to provide pion identification and tag neutral hadrons not seen by the other detectors. The same shashlik technology will be used, with an optimal segmentation devised through dedicated simulation studies.

The last component of the particle identification system will be a muon detector composed of four $6 \times 12 \mathrm{~m}^{2}$ stations, made of extruded plastic scintillator strips read out by wavelength-shifting fibers, separated by three muon filters [3].

\section{SHiP physics performance}

Thanks to the intense production of heavy hadrons in its target, SHiP will greatly improve the sensitivities of previous experiments on models with a secluded sector. At the energy accessible at the SPS, the hidden particles are predominantly produced in decays of hadrons, in particular in decays of charmed and beauty hadrons above the kaon mass, and in proton bremsstrahlung.

The SHiP physics case can be explained in terms of "portals", one per type of interaction between particles of the Hidden Sector and Standard Model particles. Each portal takes its name from the mediator of the interaction. Table 1 summarizes the portals that can be tested at SHiP, and the corresponding final states searched for. In addition to the various Hidden Sector theories, SHiP can also test predictions from supersymmetry, and directly probe for light pseudo-Goldstone bosons [2].

\subsection{Background studies}

The principal background to the hidden particle decay signal originates from the inelastic scattering of neutrinos and muons in the vicinity of the detector, producing long-lived neutral mesons. 
Table 2: Background yields for different background sources. The weight is the ratio between the generated sample and the expected yield for $N_{\text {pot }}=2 \cdot 10^{20}$. In all cases, zero events remain after applying all the selection criteria. Hence, an upper limits at $90 \% \mathrm{CL}$ is calculated as $\mathrm{UL}(90 \%)=-\ln (0.1) /$ weight. For the muon inelastic background, the upper limit is conservatively calculated by ignoring the factorizability of the veto efficiencies for the incoming muon and for the particles produced in the muon interaction. Assuming the factorizability suppresses this background by another factor $10^{3}$.

\begin{tabular}{|lcc|}
\hline Background source & Stat. weight & Expected background (UL 90\% CL) \\
\hline$v$-induced & & \\
$2.0<p<4.0 \mathrm{GeV} / \mathrm{c}$ & 1.42 & 1.62 \\
$4.0<p<10.0 \mathrm{GeV} / \mathrm{c}$ & 2.53 & 0.91 \\
$p>10 \mathrm{GeV} / \mathrm{c}$ & 3.02 & 0.76 \\
\hline $\bar{v}$-induced & & \\
$2.0<p<4.0 \mathrm{GeV} / \mathrm{c}$ & 2.41 & 0.95 \\
$4.0<p<10.0 \mathrm{GeV} / \mathrm{c}$ & 2.78 & 0.83 \\
$p>10 \mathrm{GeV} / \mathrm{c}$ & 7.23 & 0.32 \\
\hline Muon inelastic & 0.5 & 4.6 \\
\hline Muon combinatorial & - & 0.1 \\
\hline Cosmics & & \\
$p<100 \mathrm{GeV} / \mathrm{c}$ & 2.0 & 1.2 \\
$p>100 \mathrm{GeV} / \mathrm{c}$ & 1600 & 0.002 \\
\hline
\end{tabular}

Another source of background are random combinations of tracks from the residual muon flux, or other charged particles from inelastic interactions in the proximity, which enter the decay volume and together mimic signal events. Cosmic muons can contribute to both types of background, but their yield is expected to be small [3].

Careful studies using the full SHiP Monte Carlo simulation indicate that a level of background of 0.1 events for 5 years of data taking is achievable, thanks to the redundant system of veto detectors. Neutrino interactions were found to take place mainly in the muon magnetic spectrometer of the tau neutrino detector, and in the entrance window and the surrounding walls of the vacuum vessel. Background arising from muon inelastic scattering consists mainly of $K_{L}, K_{S}, \Lambda$ and $\pi^{ \pm}$decays and $\gamma$ conversion, but it is efficiently tagged by the upstream vetoes and by the surround liquid scintillator tagger. Combinatorial muons are randomly distributed over the duration of the whole spill: this background is minimized by the use of coincidence requirements based on the timing detector. Finally, the topology of events due to cosmic rays is such that already simple selection criteria (pointing and vertex quality) are able to eliminate this background.

In general, the interaction products do not point at the target, do not have a reconstructed vertex inside the decay volume, and have very poor track quality. Loose track and vertex quality requirements, together with the online preselection operated by the veto detectors, allow to reject the totality of the simulated background-induced candidate events for all background sources. This allows to set the conservative limits summarised in Table 2. The set of selections applied is highly redundant and can be trimmed down to study specific channels.

\subsection{SHiP sensitivity}

An extensive review of the sensitivities that SHiP can reach in all the probed models was published in [2]. This section summarizes, as an example, the physics reach in a specific model of 

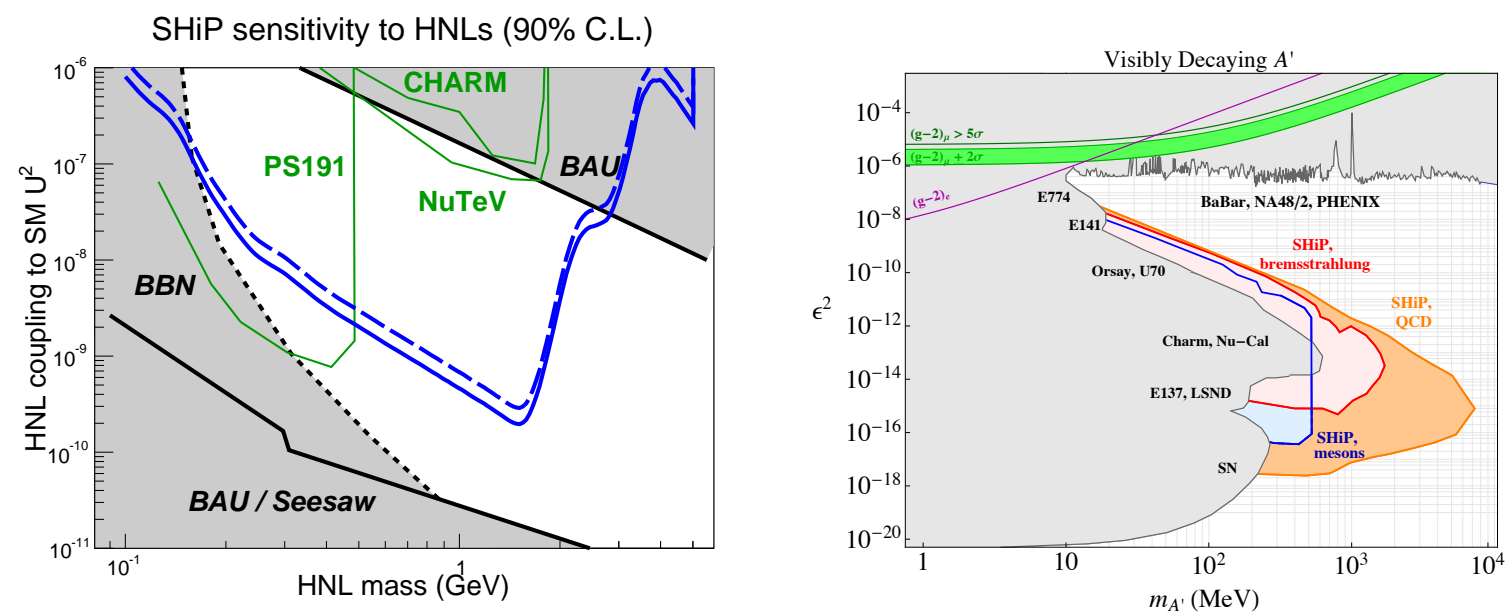

Figure 2: Left: SHiP's discovery potential in the parameter space of the $v$ MSM, for a level of background of 0.1 (continuous line) and of 10 (dashed line) events in 5 years. Right: SHiP's sensitivity to Hidden Photons.

the neutrino portal.

Previous experiments already explored part of the HNL parameter space: PS191 ruled out the $v$ MSM allowed region below the kaon mass, while CHARM and NuTeV covered couplings larger than $10^{-7}$ for masses up to $2 \mathrm{GeV}$. The SHiP experiment will be capable of extending previous searches by several orders of magnitude in the region between the kaon and the $D$ meson mass, and to access the previously unexplored region up to the $B$ meson mass. Figure 2 (left) shows the exclusion limits that can be established by SHiP at 90\% C.L. if no events are observed [3].

As a further example of SHIP's capability to shed light on the Hidden Sector, Figure 2 (right) shows SHiP's sensitivity to dark photons, gauge bosons of a minimalistic New Physic models with a broken $U(1)^{\prime}$ symmetry in the dark sector. The boundaries set by previous experiments and cosmological observations can be extended by more than one order of magnitude in mass [2].

\section{References}

[1] R. Acquafredda et al. "The OPERA experiment in the CERN to Gran Sasso neutrino beam". In: JINST 4 (2009), P04018. DOI: 10 .1088/1748-0221/4 / 04 / P 04018.

[2] Sergey Alekhin et al. "A facility to Search for Hidden Particles at the CERN SPS: the SHiP physics case". In: (2015). arXiv: 1504.04855 [hep-ph] .

[3] M. Anelli et al. "A facility to Search for Hidden Particles (SHiP) at the CERN SPS". In: (2015). arXiv: 1504.04956 [physics.ins-det].

[4] Takehiko Asaka, Steve Blanchet, and Mikhail Shaposhnikov. "The nuMSM, dark matter and neutrino masses". In: Phys. Lett. B631 (2005), pp. 151-156. DOI: 10 . $1016 / \mathrm{j}$. physletb.2005.09.070. arXiv: hep-ph/0503065 [hep-ph] .

[5] F Hahn et al. NA62: Technical Design Document. Tech. rep. NA62-10-07. Geneva: CERN, Dec. 2010. URL: https: / / cds . cern. ch/record/1404985.

[6] Giovanni De Lellis. "SHiP: a new facility with a dedicated detector for studying tau neutrino properties and nucleon structure functions". In: EPS-HEP 2015. In preparation. 